\title{
The effect of hemodialysis on intraocular pressure
}

\author{
Ayse Ebru Bahadir Kilavuzoglu, A-F, Gurkan Yurteri², B, E, F, Nurgul Guven 3, B, E, F, \\ Savas Marsap ${ }^{3, B, E, F}$, Ali Riza Cenk Celebi ${ }^{1, C, E, F}$, Cemile Banu Cosar ${ }^{1, A, C, E, F}$ \\ ${ }^{1}$ Department of Ophthalmology, Acibadem University School of Medicine, Istanbul, Turkey \\ ${ }^{2}$ Internal Medicine Clinic, Memorial Sisli Hospital, Istanbul, Turkey \\ ${ }^{3}$ Rentek Dialysis Center, Istanbul, Turkey \\ A - research concept and design; $B$ - collection and/or assembly of data; $C$ - data analysis and interpretation; \\ $D$ - writing the article; $E$ - critical revision of the article; $F$ - final approval of the article
}

\section{Address for correspondence \\ Ayse Ebru Bahadir Kilavuzoglu \\ E-mail: ebrubahadir@gmail.com \\ Funding sources \\ None declared \\ Conflict of interest \\ None declared \\ Acknowledgements \\ Scott B. Evans (language editing).}

Received on May 24, 2016

Reviewed on July 28, 2016

Accepted on January 3, 2017

DOI

10.17219/acem/68234

\section{Copyright}

Copyright by Author(s)

This is an article distributed under the terms of the

Creative Commons Attribution Non-Commercial License

(http://creativecommons.org/licenses/by-nc-nd/4.0/)

\section{Abstract}

Background. The effect of hemodialysis (HD) on intraocular pressure (IOP) has been investigated before, but there is a lack of consensus. Clinicians dealing with renal failure patients are interested in the potential negative effects of HD on IOP and the course of glaucoma.

Objectives. The aim of this study was to investigate the effects of HD on IOP in patients with end-stage renal disease.

Material and methods. This prospective study included 106 patients who were receiving outpatient hemodialysis. Patient history of systemic and ophthalmologic conditions was recorded. Serum osmolality $(\mathrm{mOsm})$, blood urea nitrogen (BUN), blood glucose (BG), bicarbonate (BC), and hematocrit (Hct) levels at the start of HD (pre-HD), at the end of HD (end-HD), and 30 min after HD (post-HD) were measured. Systolic and diastolic blood pressures (SBP and DBP) and IOP were measured at pre-HD, 1-hour intervals during $H D$, end-HD, and post-HD.

Results. A significant decrease in m0sm and BUN and a significant increase in BG, BC, and Hct levels were observed at end-HD ( $p<0.05)$. Mean IOP was $16.71 \pm 2.51 \mathrm{~mm} \mathrm{Hg}$ at pre-HD, $15.52 \pm 3.18 \mathrm{~mm} \mathrm{Hg}$ at end$H D$, and $15.23 \pm 2.73 \mathrm{~mm} \mathrm{Hg}$ at post-HD ( $p=0.001 ; F=4.439)$. Post-HD SBP and DBP were significantly lower than at pre- $\mathrm{HD}(\mathrm{p}<0.001)$. There was a positive correlation between the change in $10 \mathrm{P}$ and the change in mOsm and the change in BUN at end- $\mathrm{HD}(r=0.315, p=0.004$; and $r=0.279, p=0.012$, respectively).

Conclusions. IOP decreased significantly during HD in this study. Additional research on the effects of the change in blood parameters and ocular perfusion pressure on IOP and optic nerve perfusion during $H D$ is recommended.

Key words: glaucoma, hemodialysis, intraocular pressure 


\section{Introduction}

Glaucoma is an optic neuropathy with multifactorial etiology. While the etiological mechanism is not precisely known, elevated intraocular pressure (IOP) remains the focus of its treatment; however, some glaucoma patients continue to experience optic nerve damage despite decreased IOP. ${ }^{1,2}$ Altered ocular blood flow was reported to accelerate the development and progression of the disease in such patients; as such, not only elevated IOP, but also medical conditions that negatively affect ocular blood flow will obviously cause ischemia and reperfusion damage in glaucoma patients and in individuals prone to glaucoma. ${ }^{3-5}$

Hemodialysis (HD) is an important component of the treatment of end-stage renal disease (ESRD). Some studies have reported the effects of HD on IOP, but the findings are inconsistent. It was reported that IOP increases in response to HD, whereas it was also reported that IOP decreases or does not change during or after HD. ${ }^{6-11}$ The present study aimed to investigate the effect of HD on IOP in ESRD patients. To the best of our knowledge this is, to date, the largest prospective study on the effect of HD on IOP in ESRD patients.

\section{Material and methods}

\section{Study design and the selection of study subjects}

This prospective study included 106 patients (51 females and 55 males) who were receiving HD and met the inclusion criteria. The inclusion criteria were a negative history of glaucoma, and a healthy cornea. The exclusion criteria were IOP $>21 \mathrm{~mm} \mathrm{Hg}$ at the beginning of HD, and optic nerve abnormalities suggesting glaucoma (cupdisc ratio $>0.3$; cup-disc asymmetry between the eyes). Refractive errors, uveitis, and diabetic retinopathy were not considered as exclusion criteria unless they caused high IOP. The study was conducted in accordance with the tenets of the Declaration of Helsinki, and the institutional review board approved the study protocol. All the patients provided written informed consent.

\section{Measurements}

A Braun Dialog Hemodialysis System (Braun, Melsungen, Germany) was used for HD treatment. This system is part of a completely new generation of dialysis systems aimed at delivering optimum care with highest efficiency. It uses the principles of spectroscopy to determine the reduction in the molar concentration of urinary excreted substances in the dialyzate drain, and it enables measurement in the used dialyzate. All patients underwent 4-hour HD sessions 3 days per week. Patient body weight, and history of diabetes mellitus, hypertension, asthma, renal diseases, eye diseases, and medications were recorded. Blood samples were collected at the start of HD (pre-HD), at the end of HD (end-HD), and $30 \mathrm{~min}$ after HD (post-HD) to measure serum osmolality (mOsm), blood urea nitrogen (BUN), blood glucose (BG), bicarbonate (BC), and hematocrit (Hct). Systolic and diastolic blood pressure (SBP and DBP) and IOP were measured at pre-HD, at 1-hour intervals during HD ( $1^{\text {st }}$ hour, $2^{\text {nd }}$ hour, and $3^{\text {rd }}$ hour $)$, at end-HD ( $4^{\text {th }}$ hour $)$, and at post-HD. Pre-HD blood pressure and IOP measurements were performed after 15 min of rest, before the HD needle was inserted. Blood pressure measurement using a sphygmomanometer was always performed before IOP measurement. IOP measurement was performed using an Icare Pro tonometer (Icare, Tiolat Oy, Helsinki, Finland), with patients in the supine position. The recorded IOP was the mean of 5 successive measurements.

\section{Statistical analysis}

Statistical analysis was performed using SPSS v. 17.0 for Windows (SPSS Inc., Chicago, USA). Descriptive data is expressed as an arithmetical mean \pm SD. The independent samples t-test was used to compare the patients' right and left eyes. The paired samples t-test and oneway ANOVA for repeated measures were used to evaluate the changes in blood parameters, IOP, SBP and DBP, and the effect of systemic $\beta$-blockers on IOP change. Pearson correlation coefficient was used to determine correlations between the changes in blood parameters, IOP, SBP, and DBP. At the $95 \%$ CI, p-values $<0.05$ were accepted as statistically significant.

Table 1. mOsm, BUN, BG, BC, and Hct levels

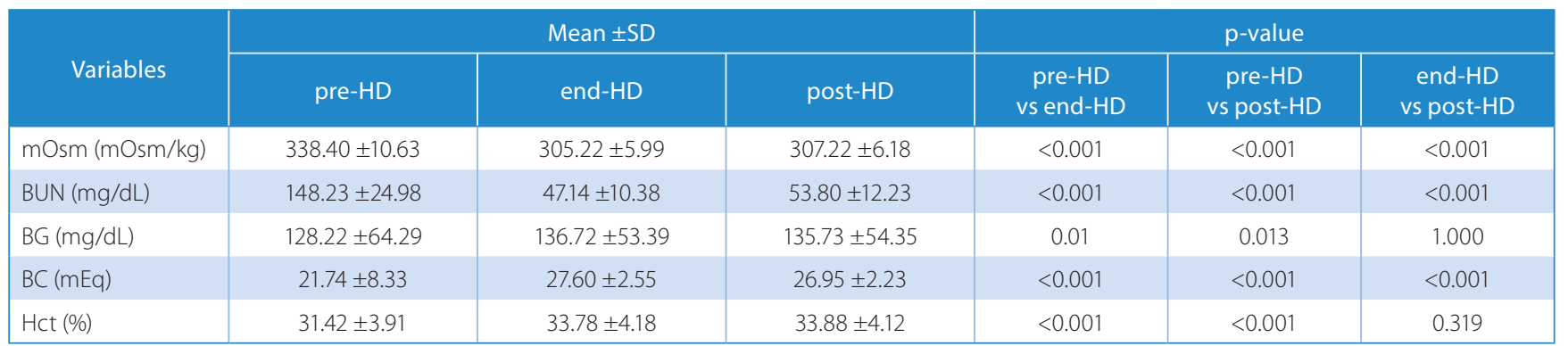

mOsm - serum osmolality; BUN - blood urea nitrogen; BG - blood glucose; BC - bicarbonate; Hct - hematocrit; pre-HD - at the start of hemodialysis; end-HD - at the end of hemodialysis; post-HD - 30 min after hemodialysis. 


\section{Results}

Mean body weight before HD was $70.22 \mathrm{~kg}$ (range: $42-$ $103.5 \mathrm{~kg}$ ) and mean age of the patients was $61.65 \pm 14.39$ years (range: 24-91 years). In all, 21 (20.3\%) patients had a history of diabetes mellitus, 80 (77.7\%) had a history of hypertension, 89 (88.1\%) had a history of renal disease (such as kidney stone, polycystic kidney disease, glomerulonephritis, etc.), and 18 (17\%) had a history of diabetic retinopathy. Among the patients, $53(50 \%)$ were using systemic $\beta$-blockers, 38 (35.8\%) were using Ca-channel blockers, and 12 (11.3\%) were using angiotensin converting enzyme inhibitors. None of the patients reported using any eye drops. Pre-HD, endHD and post-HD mOsm, BUN, BG, BC, and Hct are shown in Table 1 and Fig. 1. The values of mOsm and BUN were significantly lower, and BG, BC, and Hct were significantly higher at end-HD and post-HD, as compared to pre-HD ( $\mathrm{p}<0.05$ ). Moreover, mOsm and BUN were significantly lower at end-HD than at post-HD, and BC was significantly higher at end-HD than at post-HD ( $\mathrm{p}<0.001)$ (Table 1). There was no significant difference in IOP between right and left eyes; therefore, only right eye measurements were used for further analysis ( $\mathrm{p}>0.05)$.

IOP, SBP, and DBP measurements obtained at pre$\mathrm{HD}, 1$-hour intervals during $\mathrm{HD}$, end-HD, and post-HD are shown in Table 2 and Fig. 2. As compared to preHD, IOP decreased significantly at the $2^{\text {nd }}$ hour of HD ( $\mathrm{p}=0.009)$, end-HD ( $\mathrm{p}=0.042)$, and post-HD ( $\mathrm{p}=0.001)$. Additionally, IOP was lower at post-HD than at end-HD, but not significantly. Systemic $\beta$-blockers did not affect the changes in IOP $(p=0.443)$. SBP was significantly lower at the $2^{\text {nd }}$ hour of HD ( $\left.<<0.001\right)$, and DBP was significantly lower at the $1^{\text {st }}$ hour of $\mathrm{HD}(\mathrm{p}=0.046)$, as compared to preHD. Among the 106 patients, 105 had SBP $\geq 80 \mathrm{~mm} \mathrm{Hg}$ and 104 had DBP $\geq 50 \mathrm{~mm} \mathrm{Hg}$ during HD, at end-HD, and at post-HD. SBP and DBP increased from end-HD to post$\mathrm{HD}$, but not significantly $(\mathrm{p}>0.05)$.

Among all the evaluated measurements, there was a positive correlation between the IOP change and the mOsm and BUN change at end-HD $(r=0.315, \mathrm{p}=0.004 ; \mathrm{r}=0.279$, $\mathrm{p}=0.012$, respectively). In addition, there was a negative correlation between the change in $\mathrm{DBP}$ and the change in IOP between end-HD and post-HD $(\mathrm{r}=-0.255, \mathrm{p}=0.036)$.

\section{Discussion}

The potential effects of HD on IOP prompt clinicians who treat ESRD patients with glaucoma to considering the potential negative effects of HD on IOP and the course of glaucoma, because the deterioration of hydrodynamic parameters and blood constituent levels during dialysis are common in such patients. Altered ocular blood flow negatively affects the pathophysiology of glaucoma. ${ }^{3-5}$ In a population-based study on adults in Malaysia, chronic kidney disease was associated with elevated IOP. ${ }^{12}$ During HD, abrupt changes in blood parameters can affect the osmotic gradients between body compartments, leading to changes in IOP; thus, if HD adversely affects IOP in ESRD patients without glaucoma, those with glaucoma will not only have higher IOP, but the optic nerve may also be damaged due to hydrodynamic changes and altered ocular blood flow during HD.

The relationship between HD and IOP was first evaluated in 1964, yet there remains a lack of consensus about the effects of HD on IOP. ${ }^{6}$ It is difficult to compare findings from various studies because of the differences in IOP measurement techniques, and the ocular and non-ocular parameters investigated. Many published studies reported changes in IOP based on measurements obtained using a Goldmann applanation tonometer. ${ }^{7,9,11,13,14}$ Patients must be seated when using a Goldmann applanation tonometer. The reduction in blood volume that occurs during HD can cause hypotension and any movement during HD can cause blood pressure variation, all of which can indirectly affect IOP. ${ }^{15}$ In the present study, all measurements were made with patients in the supine position, eliminating the need for mobilization.

Some earlier studies reported that IOP increases during HD due to a decrease in mOsm. ${ }^{6,7}$ Accordingly, a rapid decrease in mOsm during HD results in an osmotic gradient and causes fluid shift from blood to the eyes, causing an increase in IOP. If there is obstruction in the outflow facility (such as anterior synechia or narrow angle), the increase in IOP is apparent, but if the outflow pathway for the aqueous humor is not obstructed, there is only a minimal increase in IOP. Sitprija et al. reported a mean rise in IOP of $5.9 \mathrm{~mm} \mathrm{Hg}$ in patients undergoing HD. ${ }^{6}$

Table 2. IOP, SBP, and DBP measurements during HD

\begin{tabular}{|c|c|c|c|c|c|c|c|}
\hline \multirow{2}{*}{ Variables } & \multicolumn{6}{|c|}{ Mean \pm SD } & \multirow{2}{*}{ p-value } \\
\hline & pre-HD & $1^{\text {st }}$ hour & $2^{\text {nd }}$ hour & $3^{\text {rd }}$ hour & end-HD & post-HD & \\
\hline IOP (mm Hg) & $16.71 \pm 2.51$ & $15.78 \pm 3.12$ & $15.42 \pm 3.28^{a}$ & $15.78 \pm 3.53$ & $15.52 \pm 3.18^{a}$ & $15.23 \pm 2.73^{a}$ & $\begin{array}{c}0.001 \\
(F=4.439)\end{array}$ \\
\hline SBP $(\mathrm{mm} \mathrm{Hg})$ & $132.01 \pm 22.29$ & $129.64 \pm 21.84$ & $123.44 \pm 19.23^{a, b}$ & $120.37 \pm 20.08^{a}$ & $116.02 \pm 20.23^{a}$ & $118.05 \pm 19.08^{a}$ & $\begin{array}{c}<0.001 \\
(F=23.096)\end{array}$ \\
\hline $\mathrm{DBP}(\mathrm{mm} \mathrm{Hg})$ & $75.37 \pm 10.88$ & $73.37 \pm 10.28^{a, b}$ & $72.03 \pm 9.85^{a}$ & $69.74 \pm 10.28^{a}$ & $68.71 \pm 10.67^{a}$ & $70.04 \pm 9.51^{\mathrm{a}}$ & $\begin{array}{c}<0.001 \\
(F=14.091)\end{array}$ \\
\hline
\end{tabular}

p - represents significance for one-way ANOVA for repeated measures; IOP - intraocular pressure; SBP - systolic blood pressure; DBP - diastolic blood pressure; HD - hemodialysis; pre-HD - at the start of hemodialysis; end-HD - at the end of hemodialysis; post-HD - 30 min after hemodialysis; a a statistically significant change compared with pre-HD measurements; ${ }^{b}$ a statistically significant change compared with the previous measurement. 


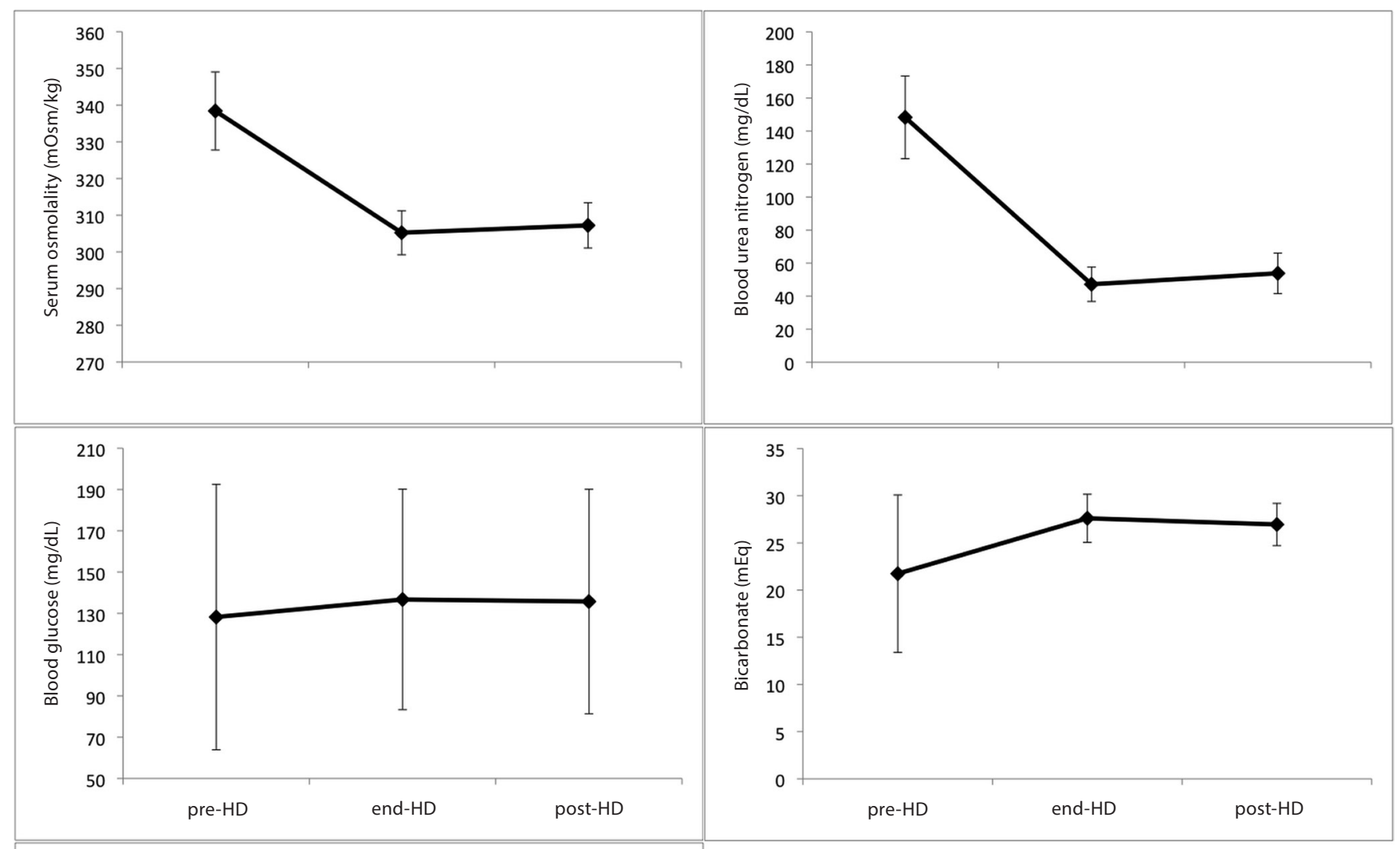

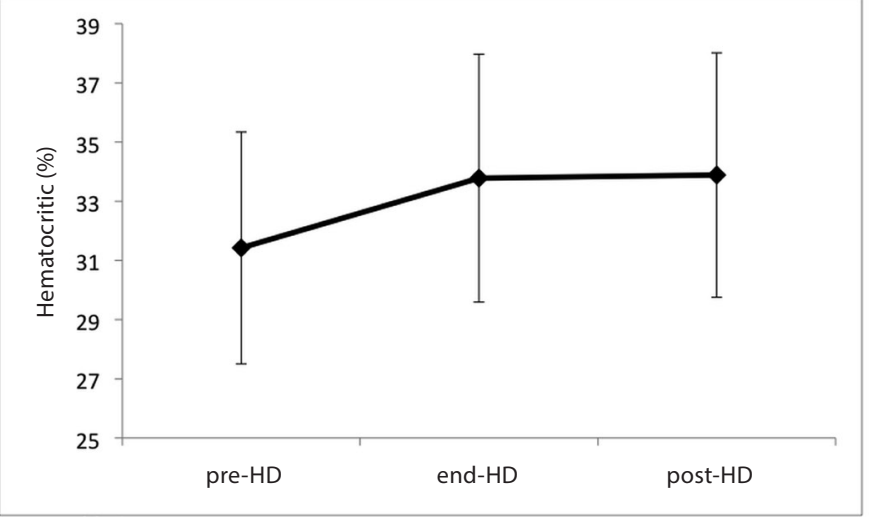

When compared with the previously reported marked increases in IOP during HD, Leiba et al. reported that the slight increase in IOP $(0.35 \mathrm{~mm} \mathrm{Hg})$ in their study was due to improved dialysis techniques. ${ }^{7}$

Some studies reported that there is no correlation between mOsm and IOP, while others reported no change in IOP during HD. ${ }^{10,11,13,16}$ Tokuyama et al. observed a decrease in IOP $(-1.8 \mathrm{~mm} \mathrm{Hg})$ and plasma osmolarity following HD, but not a significant correlation between IOP and plasma osmolarity; however, they did note a correlation between IOP and plasma colloid osmotic pressure $(r=-0.510, p=0.0012)$ and a change in body weight $(r=0.534, p=0.0006) .{ }^{9}$ According to Fauchald, plasma colloid osmotic pressure is critical to hydrodynamic changes during HD. ${ }^{17}$ The removal of water during HD decreases plasma volume and the concentration of plasma proteins increase; this increase in colloid osmotic pressure draws water from the aqueous humor into plasma. ${ }^{9,16}$
Fig. 1. Mean mOsm, BUN, BG, BC, and Hct at pre-HD, end-HD, and post-HD

mOsm - serum osmolality; BUN - blood urea nitrogen; BG - blood glucose; $\mathrm{BC}$ - bicarbonate; Hct - hematocrit; pre-HD - at the start of hemodialysis; end-HD - at the end of hemodialysis; post-HD - 30 min after hemodialysis.

In the present study there was a significant decrease in IOP during $\mathrm{HD}$ (from $16.71 \pm 2.51$ at pre-HD to $15.52 \pm 3.18$ at end$\mathrm{HD}$ ), which might have been due to the improvement in HD techniques since the time the earlier studies were performed. Slower HD may prevent abrupt changes in mOsm, and fluid shift to the eyes may be limited. Mean mOsm change was $-8.30 \pm 1.98 \mathrm{mOsm} \cdot \mathrm{kg}^{-1} \cdot \mathrm{h}^{-1}$ in the present study. Sitprija et al. reported an increase in IOP when mean mOsm change was $-11 \mathrm{mOsm} \cdot \mathrm{L}^{-1} \cdot \mathrm{h}^{-1} \cdot{ }^{6}$ During $\mathrm{HD}$, an increase in colloid osmotic pressure draws water from the aqueous humor into plasma. ${ }^{9,16}$ In the present study, the effect of elevated colloid osmotic pressure on IOP might have been greater than the effect of the decrease in mOsm on IOP, which is why HD led to lower IOP values. Despite the reported marked rise in IOP, in the present study there was a $1.2 \mathrm{~mm} \mathrm{Hg}$ of IOP decrease during HD, which is also comparable with the study conducted by Tokuyama. ${ }^{6,9} \mathrm{New}$ dialysis techniques seem to prevent marked increases in IOP during HD. 


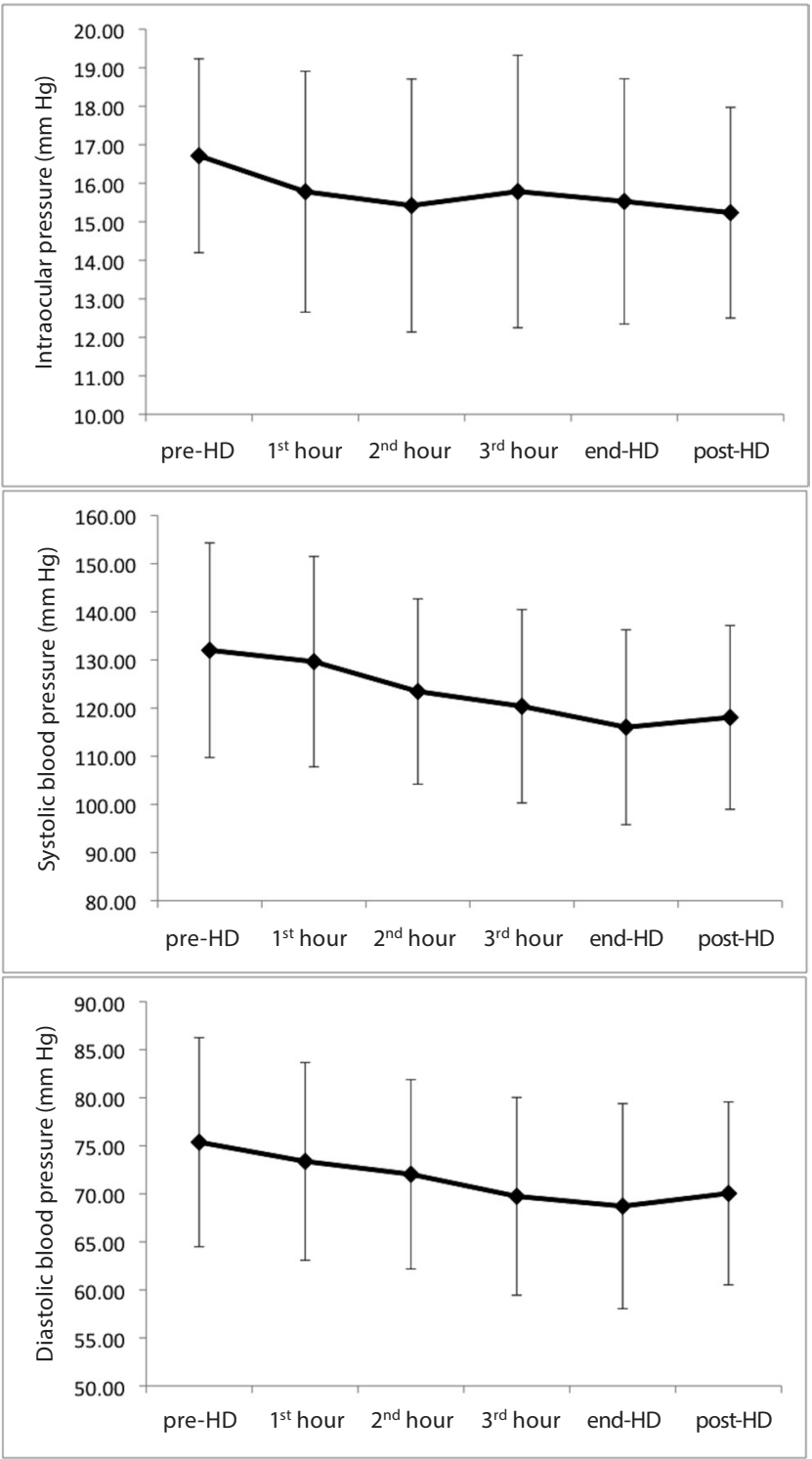

Fig. 2. Mean IOP, SBP, and DBP measurements during HD

IOP - intraocular pressure; SBP - systolic blood pressure; DBP - diastolic blood pressure; HD - hemodialysis.

During HD, a decrease in urea in the extracellular environment precedes the intracellular decrease. The difference between compartments reaches equilibrium as urea exits cells and enters extracellular fluid. This causes an increase in the serum urea level, which is known as postdialysis urea rebound (PDUR). According to Tovbin et al., PDUR was strongly correlated with intradialytic changes in IOP. ${ }^{8}$ They subtracted the urea level $1 \mathrm{~h}$ after dialysis from that measured at the end of dialysis. In the present study, BUN was measured 30 min after HD (post-HD) and the increase in BUN at post-HD was not correlated with the IOP change from end-HD to post-HD, but the decrease in BUN during HD was positively correlated with the observed decrease in IOP during HD.

As mentioned above, altered ocular blood flow accelerates the development and progression of glaucoma.
Modern HD devices, such as that used in the present study, make it possible to adjust the ultrafiltration rate according to the changes in blood pressure. ${ }^{18}$ This biofeedback system prevents hypotension via the regulation of the ultrafiltration rate and facilitates the protection of the optic nerve from the hazardous effects of hypotension, which can offer protection against ischemia and reperfusion damage in glaucoma patients and individuals prone to glaucoma. ${ }^{19}$

Aqueous humor dynamics in a glaucomatous eye differs from the normal eye. This difference is mainly related to the outflow pathways. It is reported that the resistance of the trabecular meshwork is increased in glaucomatous eyes due to an increase in extracellular matrix thickness and the deposited cochlin protein and mucopolysaccharides. ${ }^{20}$ The glycosaminoglycans in the extracellular matrix of the trabecular meshwork may generate osmotic forces and induce hydration of the trabecular meshwork. Therefore, the increased thickness in extracellular matrix in glaucomatous eyes may cause an increased resistance to aqueous outflow and eventual higher IOP. As mentioned above, the rapid decrease in mOsm with the earlier HD techniques causes a fluid shift from blood to the eyes. This would further increase IOP in glaucomatous eyes due to thicker extracellular matrix. Colloid osmotic pressure, which draws water from the aqueous humor into plasma, may counterbalance the effect of mOsm on IOP. Therefore, slower dialysis techniques seem to be beneficial for glaucomatous eyes by preventing rapid changes in serum and plasma constituents, and thereby preventing IOP rises.

ESRD patients often have chronic diseases that require the use of systemic medications. Little is known about the effects of systemic medications on IOP. A populationbased study has investigated the association between the use of common systemic medications and IOP. ${ }^{21}$ According to the results of that study, the use of systemic $\beta$-blockers and nitrates were independently associated with lower IOP. However, in the present study we did not find any effects of systemic $\beta$-blocker use on IOP decrease during HD.

The present study has some limitations. The anterior chamber was not evaluated, which would have provided some data on outflow facility; patients identified as having obstructed outflow pathways may have had findings during $\mathrm{HD}$ that differed from those of other patients. Additionally, plasma colloid osmotic pressure - possibly the primary mechanism responsible for decreasing IOP - was not measured. Another limitation of the present study is the lack of evaluation of central corneal thickness, as measured IOP has been proven to vary depending on central corneal thickness. ${ }^{22,23}$

In conclusion, the previously reported increase in IOP during HD was not observed in this study. In fact, IOP decreased significantly during HD in this study, which may be a favorable outcome for patients with ESRD and glaucoma. With continuing improvements in dialysis techniques and the advent of new devices, hydrodynamic changes during 
HD are controlled more effectively and the regulation of blood parameters and hypervolemia is improved, positively affecting IOP. We think that the observed decrease in IOP during HD in the present study was associated with a change in colloid osmotic pressure, mOsm, ocular perfusion pressure, and the outflow facility in the patients' eyes. Based on the present findings, we think additional research on the above-mentioned changes and the effects of HD on optic nerve perfusion is warranted.

\section{References}

1. Lichter PR, Musch DC, Gillespie BW, et al.; CIGTS Study Group. Interim clinical outcomes in Collaborative Initial Glaucoma Treatment Study comparing initial treatment randomized to medications or surgery. Ophthalmology. 2001;108:1943-1953.

2. Heijl A, Leske MC, Bengtsson B, Hyman L, Bengtsson, Hussein M; Early Manifest Glaucoma Trial Group. Reduction of intraocular pressure and glaucoma progression: Results from the Early Manifest Glaucoma Trial. Arch Ophthalmol. 2002;120:1268-1279.

3. Flammer J, Konieczka K, Bruno RM, Virdis A, Flammer AJ, Taddei S. The eye and the heart. Eur Heart J. 2013;34:1270-1278.

4. Mozaffarieh M, Flammer J. New insights in the pathogenesis and treatment of normal tension glaucoma. Curr Opin Pharmacol. 2013;13:43-49.

5. He Zeng, Vingrys AJ, Armitage JA, Bui BV. The role of blood pressure in glaucoma. Clin Exp Optom. 2011;94:133-149.

6. Sitprija V, Holmes JH, Ellis PP. Changes in intraocular pressure during hemodialysis. Invest Ophthalmol. 1964;3:273-284.

7. Leiba H, Oliver M, Shimshoni M, Bar-Khayim Y. Intraocular pressure fluctuations during regular hemodialysis and ultrafiltration. Acta Ophthalmol (Copenh). 1990;68:320-322.

8. Tovbin D, Belfair N, Shapira S, et al. High postdialysis urea rebound can predict intradialytic increase in intraocular pressure in dialysis patients with lowered intradialytic hemoconcentration. Nephron. 2002;90:181-187.
9. Tokuyama T, Ikeda T, Sato K. Effect of plasma colloid osmotic pressure on intraocular pressure during haemodialysis. Br JOphthalmol. 1998:82:751-753.

10. Hojs R, Pahor D. Intraocular pressure in chronic renal failure patients treated with maintenance hemodialysis. Ophthalmologica. 1997;211:325-326.

11. Pelit A, Zumrutdal A, Akova Y. The effect of hemodialysis on visual field test in patients with chronic renal failure. Curr Eye Res. 2003;26:303-306.

12. Nongpiur ME, Wong TY, Sabanayagam C, Lim SC, Tai ES, Aung T. Chronic kidney disease and intraocular pressure: The Singapore Malay Eye Study. Ophthalmology. 2010;117:477-483.

13. Gafter U, Pinkas M, Hirsch J, Levi J, Savir H. Intraocular pressure in uremic patients on chronic hemodialysis. Nephron. 1985;40:74-75.

14. Masuda H, Shibuya Y, Ohira A. Markedly increased unilateral intraocular pressure during hemodialysis in a patient with ipsilateral exfoliative glaucoma. Am J Ophthalmol. 2000;129:534-536.

15. Klein $B E$, Klein R, Knudtson MD. Intraocular pressure and systemic blood pressure: Longitudinal perspective: The Beaver Dam Eye Study. Br J Ophthalmol. 2005;89:284-287.

16. Samsudin A, Mimiwati Z, Soong T, Fauzi MS, Zabri K. Effect of haemodialysis on intraocular pressure. Eye (Lond). 2010;24:70-73.

17. Fauchald P. Transcapillary colloid osmotic gradient and body fluid volumes in renal failure. Kidney Int. 1986;29:895-900.

18. Palmer BF, Henrich WL. Recent advances in the prevention and management of intradialytic hypotension. J Am Soc Nephrol. 2008;19:8-11.

19. Memarzadeh F, Ying-Lai M, Chung J, Azen SP, Varma R; Los Angeles Latino Eye Study Group. Blood pressure, perfusion pressure, and open-angle glaucoma: The Los Angeles Latino Eye Study. Invest Ophthalmol Vis Sci. 2010;51:2872-2877.

20. Keller KE, Acott TS. The juxtacanalicular region of ocular trabecular meshwork: A tissue with a unique extracellular matrix and specialized function. J Ocul Biol. 2013;1:3.

21. Khawaja AP, Chan MP, Broadway DC, et al. Systemic medication and intraocular pressure in a British population: The EPIC-Norfolk Eye Study. Ophthalmology. 2014;121:1501-1507.

22. Whitacre MM, Stein R. Sources of error with use of Goldmann-type tonometers. Surv Ophthalmol. 1993;38:1-30.

23. Whitacre MM, Stein RA, Hassanein K. The effect of corneal thickness on applanation tonometry. Am J Ophthalmol. 1993;115:592-596. 